\title{
Upfront Docetaxel with LH-RH Antagonist for Metastatic Hormone Sensitive Prostate Cancer Considering Epithelial to Mesenchymal Transition
}

\author{
Teiichiro Aoyagi*, Isao Kuroda, Go Nagao \\ Department of Urology, Tokyo Medical University, Ibaraki Medical Center, Ibaraki, Japan \\ Email: *aoyagite@tokyo-med.ac.jp
}

How to cite this paper: Aoyagi, T., Kuroda, I. and Nagao, G. (2020) Upfront Docetaxel with LH-RH Antagonist for Metastatic Hormone Sensitive Prostate Cancer Considering Epithelial to Mesenchymal Transition. Open Journal of Urology, 10, 284-292. https://doi.org/10.4236/oju.2020.1011033

Received: October 12, 2020

Accepted: November 10, 2020

Published: November 13, 2020

Copyright $\odot 2020$ by author(s) and Scientific Research Publishing Inc. This work is licensed under the Creative Commons Attribution International License (CC BY 4.0).

http://creativecommons.org/licenses/by/4.0/

(c) (i) Open Access

\begin{abstract}
Objective: Upfront docetaxel use for hormone naïve advanced prostate cancer is reported that it successfully delayed the progression to hormone refractory stage, though the adequate methodology to obtain the maximum effect is unclear. We investigated these issues from our experiences of upfront docetaxel use with LH-RH antagonist for metastatic hormone sensitive prostate cancer, aiming at the prevention of epithelial-mesenchymal transition (EMT) for apoptosis tolerance. Patients and Methods: Of 31 stage IV new prostate cancer patients treated with upfront docetaxel and LH-RH antagonist (Degarelix), 25 patients who could be followed more than 12 months (mean 36.2 months) were analyzed. Docetaxel was used two to three courses basically $75 \mathrm{mg} / \mathrm{m}^{2}$ dose initializing two weeks after the induction of first Degarelix. Results: The clinical course was divided clearly to two groups according to prostate specific antigen (PSA) values. Of 25 patients, 12 patient's PSA did not decrease below $0.1 \mathrm{ng} / \mathrm{ml}$ within 6 months (group A) and gradually rose afterwards. PSA in another 13 patients (group B) decreased below 0.1 within 6 months and kept below 0.1 during the follow up period. Although statistically not significant, the initial group A's PSA was higher than group B's (average 1308 and $353 \mathrm{ng} / \mathrm{ml}$ ), however, number of metastasis, Gleason sum, and bone metastatic extent of disease showed no difference between them. Among group B patients, 7 cases had only upfront docetaxel and hormonal therapy, and some of these patients showed only atrophic gland and fibrotic tissue at second prostate biopsy (specimens after more than two years of therapy), suggesting complete response. Conclusion: Our study suggested that PSA value at 6 months may predict the outcome of whole therapy. Patients showing PSA less than $0.1 \mathrm{ng} / \mathrm{ml}$ at 6 months and requiring
\end{abstract}


no therapy other than docetaxel and hormone may be induced to complete response. Upfront docetaxel with LH-RH antagonist may prevent EMT for obtaining apoptosis tolerance, in case the patient does not have the castration-resistant clone at the beginning of the therapy (group B).

\section{Keywords}

Docetaxel, Prostate Cancer, Epithelial to Mesenchymal Transition, Degarelix

\section{Introduction}

Although early detection of non-metastatic prostate cancer improved overall and disease specific survival for all prostate cancer patients, no apparent evidence of survival improvement was shown for de novo metastatic prostate cancer patients [1]. The GETUG-AFU-15 [2], CHAARTED [3] and STAMPEDE NCT00268476 [4] RCT studies proved the efficacy of upfront docetaxel use for metastatic hormone-sensitive prostate cancer (mHSPC) in median progression free survival and/or median overall survival. Following the evidences of these studies, upfront docetaxel therapy is becoming a standard care for patients with mHSPC [5] [6]. However, the best timing to start docetaxel and/or the duration of chemotherapy have not to be cleared [7] [8]. The reason why androgen deprivation therapy (ADT) must be continued eternally is said that small numbers of cancer cells obtain apoptosis tolerance at the beginning of the ADT, and keep alive at cell arrest phase as long as ADT continues, while most of the hormone-sensitive cancer cells go into apoptosis [9]. And these remaining cells have a chance to get an ability to proliferate even in low hormonal environment in some future, and become castrate resistant prostate cancer [10]. The LHRH-antagonist, Degarelix is proved to cut androgen to castrate level as early as surgical castration [11]. We use docetaxel as tubulin-targeting chemotherapy at the beginning of Degarelix hormonal therapy aiming at "no cancer cells" obtaining apoptosis tolerance. We here report an interesting result that may lead to more effective upfront docetaxel use as a methodology.

\section{Patients and Methods}

Of 31 stage IV new prostate cancer patients during the period of Oct. 2014 to Oct. 2019, treated with upfront docetaxel and LH-RH antagonist (Degarelix), 25 patients who could be followed more than 12 months (mean 36.2 months) were analyzed. Patients who had been treated surgically or radiologically as localized prostate cancer and advanced thereafter were excluded, because mostly they had already been hormonally treated when prostate specific antigen (PSA) recurrence. No age limitation was provided if the patient could approve chemotherapy. Docetaxel was used two to three courses monthly basically $75 \mathrm{mg} / \mathrm{m}^{2}$ dose initializing two weeks after the induction of first Degarelix. As this study was not 
a controlled study but an observational study, for the patients underwent upfront docetaxel and Degarelix, attending physician added other drugs and therapies afterwards according to necessity, such as regional irradiation, abiraterone, enzalutamide and cabazitaxel. Patients, whose initial chief complaint was progressive limb paralysis due to vertebral metastasis, underwent irradiation for the metastatic site and Degarelix as a first therapy. Transrectal prostate biopsy was performed for all patients before treatment, and second prostate biopsy was also made for selected patients showing good clinical course for more than two years. Statistical analysis was made by Student's t-test for the initial PSA values between A and B. This study was approved by the hospital's IRB committee (Institutional Review Board of Tokyo Medical University, Ibaraki Medical Center, approved \# 14 - 39) and all the attendees accepted this study and report by signature.

\section{Results}

The clinical course of this therapy was divided clearly into two groups according to PSA values. Of 25 patients, 12 patient's PSA did not decrease below $0.1 \mathrm{ng} / \mathrm{ml}$ within 6 months and gradually rose afterwards. These patients were categorized as group A. PSA in another 13 patients decreased below 0.1 within 6 months and kept below 0.1 during the follow up period. These patients were categorized as group B (Figure 1(a), Figure 1(b)). Although statistically not significant, the initial group A's PSA were higher than group B's (average 1308 and $353 \mathrm{ng} / \mathrm{ml}$ ), however, number of metastasis, Gleason sum, and bone metastatic extent of disease showed no difference between them (Table 1). Though group A patients had various additional therapy after PSA failure by physician's decision, among group B patients, seven patients only underwent hormonal and upfront docetaxel therapy. Of those, two patients showed only atrophic gland and fibrotic tissue at second prostate biopsy after more than two years follow up, suggesting complete response only by hormonal and upfront docetaxel therapy (Figure 2(a), Figure 2(b)).

\section{Discussion}

The evolving treatment progress have occurred for metastatic prostate cancer by the development of new generation anti-androgens [12], abiraterone acetate which inhibit adrenal testosterone biosynthesis more effectively than non-specific CYP 17 inhibitor such as ketoconazole and aminoglutethimide [13], and taxanes that bind beta-tubuline and stabilize the microtubule cytoskeleton leading an apoptotic cell death [14]. These agents have been used for hormone refractory advanced prostate cancer, and attribute to improve overall survival and progression free survival. However, in recent years, upfront use of these agents for hormone-sensitive prostate cancer, showed significant retardation of the development to hormone-refractory state [15]. Usefulness of upfront docetaxel for mHSPC was proved by randomized prospective studies such as GETUG-AFU-15 
NCT00104715 [2], CHAARTED NCT00309985 [3], STAMPEDE NCT00268476 [4], though the best timing and duration of additional chemotherapy have not been determined [16]. As a hormonal agent, LHRH agonist was mainly used for these studies. The timing to start upfront chemotherapy varied from simultaneous to several months from the beginning of hormonal therapy by these study design. We used Degarelix (LHRH-antagonist), because it was proved to reduce androgen to castrate level as early as surgical castration, and induce apoptosis simultaneously for all hormone-sensitive benign and malignant prostatic cells [11].
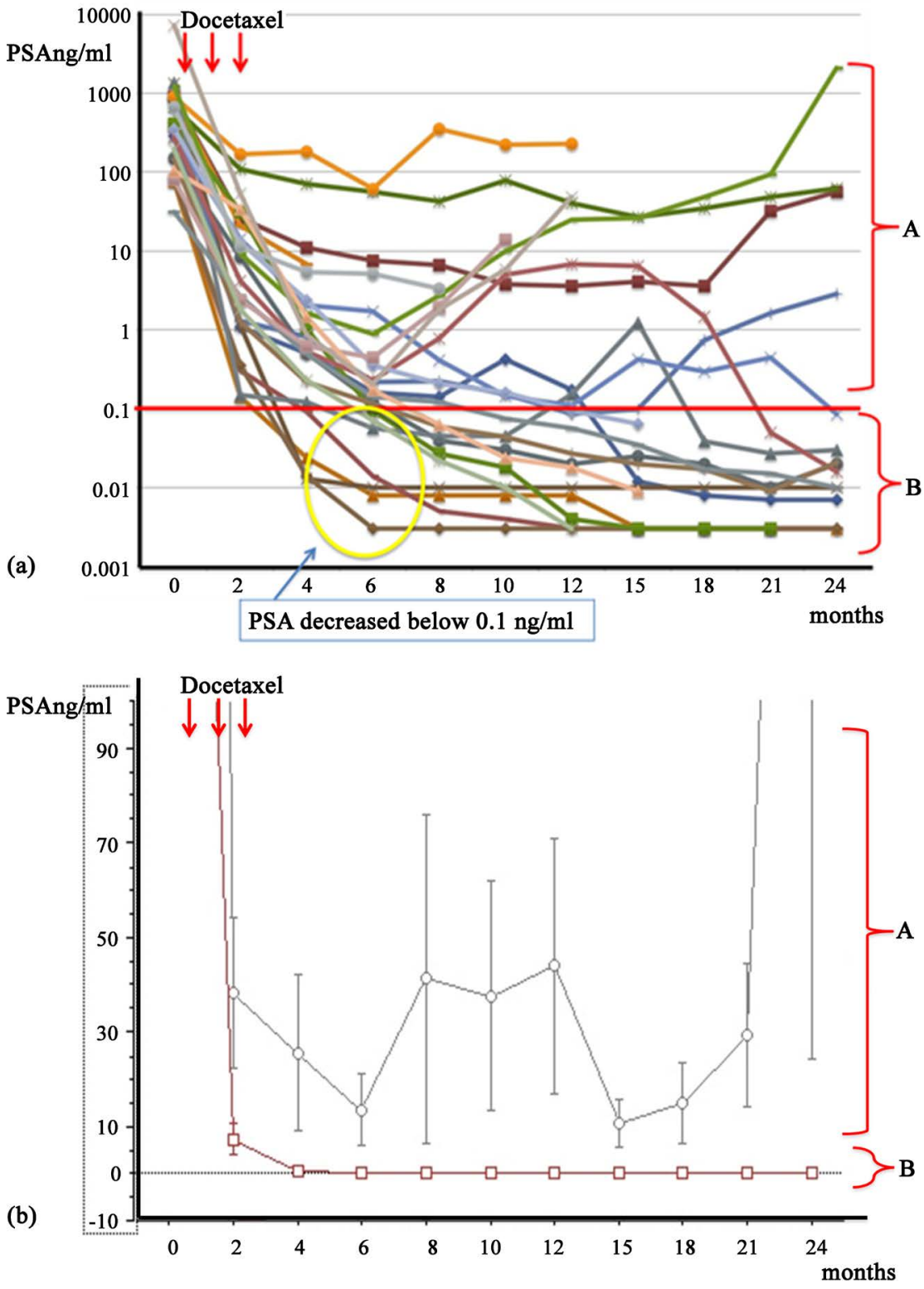

Figure 1. (a) Clinical course by PSA values of all patients are indicated in Semi-log plot. Patients whose PSA did not decrease below $0.1 \mathrm{ng} / \mathrm{ml}$ within 6 months were categorized as group A, and the others as group B. (b) Clinical course by PSA values of group average $\pm \mathrm{SD}$ in lineal plot. 
Table 1. Patient profiles of group A and B.

\begin{tabular}{|c|c|c|c|}
\hline & & A & B \\
\hline \multicolumn{2}{|c|}{ No. of patients } & 12 & 13 \\
\hline \multicolumn{2}{|c|}{ Average age (min-max)y/o } & $71.7(60-83)$ & $66.9(52-77)$ \\
\hline \multicolumn{2}{|c|}{ Initial PSA (ng/ml) } & $1308.5(80-7184)$ & $353.4(31-1371)$ \\
\hline \multirow{3}{*}{$\begin{array}{c}\text { Gleason Grade } \\
\text { Group }\end{array}$} & 3 & 0 & 1 \\
\hline & 4 & 4 & 5 \\
\hline & 5 & 7 & 6 \\
\hline Bone Mets & $1-2$ & 3 & 5 \\
\hline EOD & $3-4$ & 5 & 5 \\
\hline
\end{tabular}

No statistical differences were observed between A and B in these profiles. EOD: extent of disease.
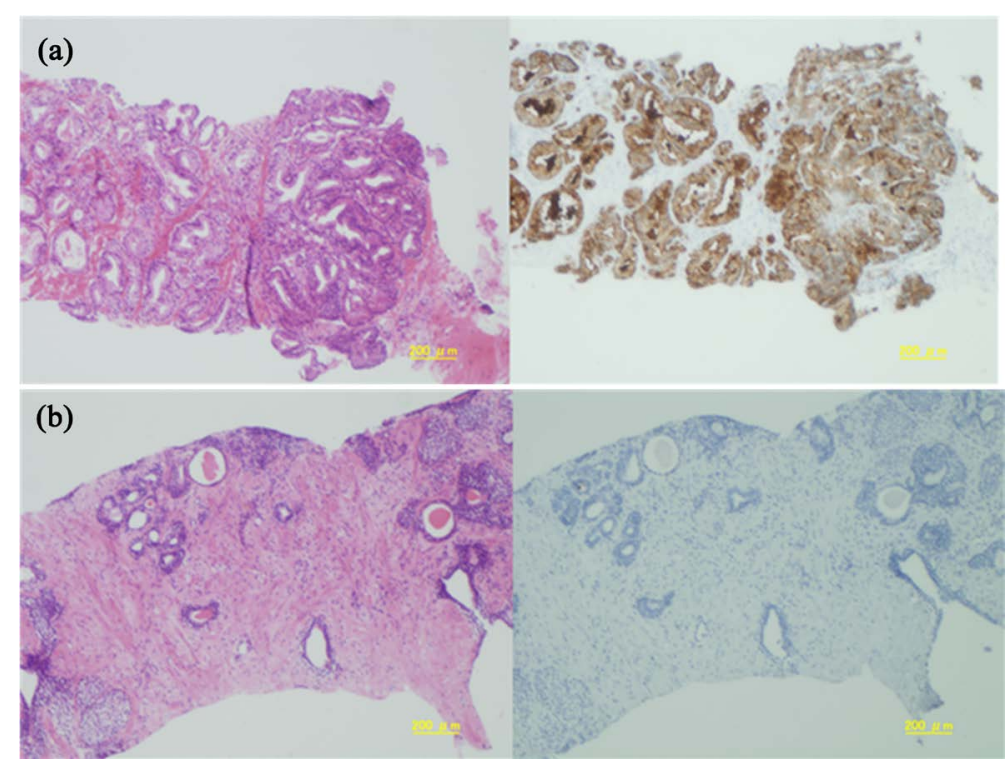

Figure 2. (a) Representative histological findings of group B patients showing initial (pre-treatment) prostate biopsy. Gleason $4+3$ adenocarcinoma observed in H \& E (left), and immunohistochemical staining for PSA (right). Reduced from 20×. (b) Second (post treatment more than two years) prostate biopsy from the same patient as Figure 2(a). Only atrophic glands with negative PSA staining (right) were shown. Reduced from $20 \times$.

The mechanism and timing of development of castration resistance are still debatable. Two models were proposed such as; "adaptation emergence" and "clonal selection". The former model suggested that prostate cancer is composed of homogenous hormone dependent cells, and castration resistance emerges through genetic/epigenetic conversion of cells from androgen-dependent to independent status. Whereas the latter model indicates that prostate cancer is composed of heterogenous major hormone dependent and minor hormone independent cells. Under an androgen-deprived environment, the castration-resistant cells are selected for survival and obtain proliferative advantage, and finally all cells composed of hormone independent [17]. Especially to the 
former adaptation emergence process, the contribution of epithelial to mesenchymal transition (EMT) has been proved in the several basic studies [18]. And for the purpose of inhibiting EMT and/or mutation of androgen receptor, microtubule-targeting agents; docetaxel is reported effective [19] [20]. We consider that docetaxel should be used at the timing of the first hormonal use, that is the timing of adaptation emergence to acquire apoptotic tolerance for hormonal therapy [21]. Eigl et al. (2005) reported an in vivo synergistic effect of taxans and ADT. They found that homogenous hormone-sensitive tumor engrafted mice receiving paclitaxel and simultaneous castration, exhibited a delayed median time to progression compared to those treated with sequential ADT and chemotherapy [22]. Our results suggested that in group A, small numbers of survived castration resistant prostate cancer, those existed at the first visit, continued proliferation after the chemotherapy as a "clonal selection". Whereas in group B, especially in patients applying no other treatment modality other than ADT and docetaxel, upfront docetaxel prevented EMT to obtain "adaptation emergence", and induced total cell kill. Second prostate biopsy for those PSA value below $0.1 \mathrm{ng} / \mathrm{ml}$ for more than two years and quitted ADT, indicated no residual cancer on specimens. This result may prove our total kill cell theory. Sasaki and Sugimura (2018) summarized the relation of prognosis between nadir PSA value and time to PSA nadir after primary ADT for advanced hormone-naïve prostate cancer. They concluded that higher PSA nadir and shorter time to PSA nadir represents poor prognostic factor, suggesting more androgen and androgen-receptor independent cancer cells at the first visit [23]. Moreover, our results suggested that the decrease of PSA less than $0.1 \mathrm{ng} / \mathrm{ml}$ within 6 months predicted good prognosis. It was presumed that rapid PSA reduction indicated high proportion of hormone-sensitive cancer cells at the first visit [23]. Group B patients were thought to have high proportion of hormone-sensitive cancer. In experimental animal study consisted all hormone-sensitive cancer cells, a closely timed sequence of chemo-hormonal therapy appeared to induce maximum synergy effect [22]. Our results might prove clinical model of Eigl's study in group B patients. According to the results of CHAARTED and GETUG-AFU15 studies, upfront docetaxel was more beneficial for high volume mHSPC rather than low volume cancer [24]. However, considering EMT, Eigl's and our results it is suggested that even for low volume mHSPC, upfront docetaxel will be beneficial. The timing of upfront docetaxel initiation should start two weeks after the first LHRH antagonist, to disturb EMT of hormone naïve cells for the acquisition of apoptosis tolerance.

\section{Conclusion}

Although the number of patients and follow up period were limited, our study suggested that PSA value at 6 months may predict the outcome of whole therapy. Patients showing PSA less than $0.1 \mathrm{ng} / \mathrm{ml}$ at 6 months and requiring no therapy other than docetaxel and hormone may be induced to complete response. Upfront docetaxel with LH-RH antagonist may prevent EMT for ob- 
taining apoptosis tolerance, in case the patient does not have the castration-resistant clone at the beginning of the therapy (group B).

\section{Conflicts of Interest}

The authors declare no conflicts of interest regarding the publication of this paper.

\section{References}

[1] Wu, J.N., Fish, K.M., Evans, C.P., White, R.W. and Dall'Era, M.A. (2014) No Improvement Noted in Overall or Cause-Specific Survival for Men Presenting with Metastatic Prostate Cancer over a 20-Year Period. Cancer, 120, 818-823. https://doi.org/10.1002/cncr.28485

[2] Gravis, G., Fizzazi, K., Joly, F., Oudard, S., Priou, F., Esterni, B., et al. (2013) Androgen-Deprivation Therapy Alone or with Docetaxel in Non-Castrate Metastatic Prostate Cancer (GETUG-AFU 15): A Randomized, Open-Label, Phase 3 Trial. The Lancet Oncology, 14, 149-158. https://doi.org/10.1016/S1470-2045(12)70560-0

[3] Sweeney, C.J., Chen, Y.H., Carducci, M., Liu, G., Jarrard, D.F., Eisenberger, M., et al. (2015) Chemohormonal Therapy in Metastatic Hormone-Sensitive Prostate Cancer. The New England Journal of Medicine, 373, 737-746. https://doi.org/10.1056/NEJMoa1503747

[4] James, N.D., Sydes, M.R., Mason, M.D., Clarke, N.W., Anderson, J., Dearnaley, D.P., et al. (2015) Docetaxel and/or Zoledronic Acid for Hormone-Naïve Prostate Cancer: First Overall Survival Results from STAMPEDE (NCT00268476). Journal of Clinical Oncology, 33, 5001. https://doi.org/10.1200/jco.2015.33.15_suppl.5001

[5] Mottet, N., van den Bergh, R.C.N., Briers, E., Bourke, L., et al. (2018) EAU-ESTROESUR-SIOG Guidelines on Prostate Cancer. 6.4.5.2 ADT Combined with Chemotherapy. 70 .

https://uroweb.org/wp-content/uploads/EAU-ESUR-ESTRO-SIOG-Guidelines-onProstate-Cancer-large-text-V2.pdf

[6] Committee for Medicinal Products for Human Use (CHMP) Opinion "TAXOTERE in Combination with Androgen-Deprovation Therapy (ADT), with or without Prednisone or Prednisolone, Is Indicated for the Treatment of Patients with Metastatic Hormone-Sensitive Prostate Cancer."

https://www.ema.europa.eu/en/documents/smop/chmp-post-authorisation-summa ry-positive-opinion-taxotere-ws-1550_en.pdf

[7] Sun, G., Zhang, X., Chen, J., Liao, B., et al. (2018) What Kind of Patients with Castration-Naïve Prostate Cancer Can Benefit from Upfront Docetaxel and Abiraterone: A Systematic Review and a Network Meta-Analysis. Urologic Oncology, 36, 505-517. https://doi.org/10.1016/j.urolonc.2018.09.005

[8] Gravis, G., Audenet, F., Irani, J., Timsit, M.O., et al. (2017) Chemotherapy in Hormone-Sensitive Metastatic Prostate Cancer: Evidences and Uncertainties from the Literature. Cancer Treatment Reviews, 55, 211-217. https://doi.org/10.1016/j.ctrv.2016.09.008

[9] Akakura, K., Bruchovsky, N., Goldenberg, S.L., Rennie, P.S., Buckley, A.R. and Sullivan, L.D. (1993) Effects of Intermittent Androgen Suppression on Amdrogen-Dependent Tumors. Apoptosis and Serum Prostate-Specific Antigen. Cancer, 71, 2782-2790.

https://doi.org/10.1002/1097-0142(19930501)71:9<2782::AID-CNCR2820710916>3. 
$\underline{0 . \mathrm{CO} ; 2-\mathrm{Z}}$

[10] Huang, Y., Jiang, X., Liang, X. and Jiang, G. (2018) Molecular and Cellular Mechanism of Castration Resistant Prostate Cancer (Review). Oncology Letters, 15, 6063-6076. https://doi.org/10.3892/ol.2018.8123

[11] Shore, N.D. (2013) Experience with Degarelix in the Treatment of Prostate Cancer. Therapeutic Advances in Urology, 5, 11-24. https://doi.org/10.1177/1756287212461048

[12] Crawford, E.D., Schellhammer, P.F., McLeod, D.G., Moul, J.W., Higano, C.S., Shore, N., et al. (2018) Androgen Receptor Targeted Treatments of Prostate Cancer; 35 Years of Progress with Antiandrogens. Journal of Urology, 200, 956-966. https://doi.org/10.1016/j.juro.2018.04.083

[13] Ryan, C.J., Smith, M.R., de Bono, J.S., Molina, A., Logothetis, C.J., de Souza, P., et al. (2013) Randomized Phase 3 Trial of Abiraterone Acetate in Men with Metastatic Castration-Resistant Prostate Cancer and No Prior Chemotherapy. New England Journal of Medicine, 368, 138-148.

[14] Darshan, M.S., Loftus, M.S., Thadani-Mulero, M., Levy, B.P., Escuin, D., Zhou, X.K., et al. (2011) Taxane-Induced Blockade to Nuclear Accumulation of the Androgen Receptor Predicts Clinical Response in Metastatic Prostate Cancer. Cancer Research, 71, 6019-6029. https://doi.org/10.1158/0008-5472.CAN-11-1417

[15] Fizazi, K., Jenkins, C. and Tannock, I.F. (2015) Should Docetaxel Be Standard of Care for Patients with Metastatic Hormone-Sensitive Prostate Cancer? Pro and Contra. Annals of Oncology, 26, 1660-1667. https://doi.org/10.1093/annonc/mdv245

[16] Shiota, M., Yokomizo, A. and Eto, M. (2016) Taxane Chemotherapy for Hormone-Naïve Prostate Cancer with Its Expanding Role as Breakthrough Strategy. Frontiers in Oncology, 5, 304. https://doi.org/10.3389/fonc.2015.00304

[17] Ahmed, M. and Li, L.C. (2013) Adaptation and Clonal Selection Models of Castration-Resistant Prostate Cancer: Current Perspective. International Journal of Urology, 20, 362-371. https://doi.org/10.1111/iju.12005

[18] Li, P., Yang, R. and Gao, W. (2014) Contributions of Epithelial-Mesenchymal Transition and Cancer Stem Cells to the Development of Castration Resistance of Prostate Cancer. Molecular Cancer, 13, 55. https://doi.org/10.1186/1476-4598-13-55

[19] Martin, S.K., Kamelgarn, M. and Kyprianou, N. (2014) Cytosleleton Targeting Value in Prostate Cancer Treatment. American Journal of Clinical and Experimental Urology, 2, 15-26. https://pubmed.ncbi.nlm.nih.gov/25374905/

[20] Zhu, M.L., Horbinski, C.M., Garzotto, M., Qian, D.Z., Beer, T.M. and Kyprianou, N. (2010) Tubulin-Targeting Chemotherapy Impairs Androgen Receptor Activity in Prostate Cancer. Cancer Research, 70, 7992-8002. https://doi.org/10.1158/0008-5472.CAN-10-0585

[21] Jaworska, D. and Szliszka, E. (2017) Targeting Apoptotic Activity against Prostate Cancer Stem Cells. International Journal of Molecular Sciences, 18, 1648. https://doi.org/10.3390/ijms18081648

[22] Eigl, B.J.C., Eggener, S.E., Baybik, J., Ettinger, S., Chi, K.N., Nelson, C., et al. (2005) Timing Is Everything: Preclinical Evidence Supporting Simultaneous Rather than Sequential Chemohormonal Therapy. Clinical Cancer Research, 11, 4905-4911. https://doi.org/10.1158/1078-0432.CCR-04-2140

[23] Sasaki, T. and Sugimura, Y. (2018) The Importance of Time to Prostate-Specific Antigen (PSA) Nadir after Primary Androgen Deprivation Therapy in Hormone-Naïve Prostate Cancer Patients. Journal of Clinical Medicine, 7, 565. 
https://doi.org/10.3390/jcm7120565

[24] Gravis, G., Boher, J.M., Chen, Y.H., et al. (2018) Burden of Metastatic Castrate Naïve Prostate Cancer Patients, to Identify Men More Likely to Benefit from Early Docetaxel: Further Analysis of CHAARTED and GETUG-AFU15 Studies. European Urology, 73, 847-855. https://doi.org/10.1016/j.eururo.2018.02.001 